\title{
The massive and evolved EBS V380 Cygni: A case of critical evolution
}

\begin{abstract}
A. Claret*
Instituto de Astrofísica de Andalucía, CSIC, Apartado 3004, 18080 Granada, Spain

Received 5 April 2002 / Accepted 27 November 2002

Abstract. The evolution and the internal structure of the double-lined eclipsing binary V380 Cyg showing apsidal motion is investigated. The system consists of an evolved massive primary with a mass of $11.1 \pm 0.5 M_{\odot}$ and a secondary which is still close to the ZAMS and has a mass of $6.95 \pm 0.25 M_{\odot}$. The core overshooting parameter $\alpha_{\text {ov }}$ was recently determined for this star as $0.6 \pm 0.1$. However, such a value was derived using error bars in the effective temperature which seem to be unrealistically small. In addition, there is no independent support for this value of $\alpha_{\text {ov }}$ either from Colour-Magnitude Diagrams of clusters or from double-lined eclipsing binaries. Therefore, we decided to compute stellar models with several values of core-overshooting parameter in order to try to disentangle this apparent disagreement. In order to compare the observations with the theoretical predictions, we decided to use the ratio of the effective temperatures, TR $=T_{\text {eff2 }} / T_{\text {effl } 1}$, rather than the effective temperatures themselves to avoid problems with calibrations and/or model atmosphere dependencies. Contrary to what has been previously established, we have found acceptable common ages for $\alpha_{\mathrm{ov}}=0.2,0.4$, and 0.6 which fit the TR, the masses, the radii and the apsidal-motion rate simultaneously. Such indetermination in $\alpha_{\mathrm{ov}}$ should not be interpretated as a negative result. Instead, it indicates that errors in $\alpha_{\text {ov }}$ cannot be determined with a precision of 0.1 if realistic observational error bars are used; we are dealing with an EBS in a very critical phase of evolution. Moreover, these new inferred values of $\alpha_{\mathrm{ov}}$ imply that the dependency of convective core overshooting on the stellar mass, if it exists, is more uncertain and less pronounced than previous investigations suggested.
\end{abstract}

Key words. stars: binaries: close - stars: evolution - stars: early-type - stars: fundamental parameters

\section{Introduction}

The ability of double-lined eclipsing binary systems (DLEBS) to provide accurate stellar masses and radii with mean errors around $1-2 \%$ is widely known. However, there are also DLEBS, such as V380 Cyg, $\alpha$ Vir and V1765 Cyg, which were considered problematic not only regarding the determination of their absolute dimensions but also regarding their internal structure. The three mentioned systems were used in the past to check the stellar internal structure predictions for evolved binaries through the apsidal-motion test, and the corresponding discrepancies between the observed and theoretical apsidal-motion rates defined by $\delta \log k_{2} \equiv \log k_{2 \mathrm{obs}}-\log k_{2 \text { theo }}$ were very conspicuous. In fact, they showed the largest deviations. For quite some time, the work carried out by several investigators indicated that the theoretical stellar models predicted lower grades of mass concentration than what was observed for real stars. With the improvement of both low- and high-temperature opacities (Huebner et al. 1977; Iglesias \& Rogers 1996; Alexander \& Ferguson 1994; Kurucz 1994) and of nuclear reaction rates (Caughlan \& Fowler 1988), the situation has now improved drastically, making a more severe comparison with observational data finally possible. Consequently, the theoretical apsidal-motion rates were recently revised by

\footnotetext{
* e-mail: claret@iaa.es
}

Claret \& Willems (2002), who introduced accurate and updated observational data as well as theoretical improvements such as recent opacities, stellar rotation, and dynamic tides. The resulting comparison between theory and observations resulted in a much better inter-agreement than ever before.

Meanwhile, the status of the three mentioned anomalous systems did not change much for several years. The required levels of observational accuracy for the absolute dimensions and the apsidal-motion rate have been approached only in the case of V380 Cyg (Guinan et al. 2000). In the latter paper, the masses, radii, effective temperatures and apsidal-motion rate were derived more accurately than previously. Combining these elements with stellar models, the convective overshooting parameter was derived to be $\alpha_{\mathrm{ov}}=0.6 \pm 0.1$. However, such a value of $\alpha_{\text {ov }}$ seems to be too high and in disagreement with those derived for a significant sample of DLEBS or from Color-Magnitude Diagrams of clusters, which are in the range 0.2-0.3. In addition, the error bars in the effective temperatures and in the distance ( $4 \%$ in $1000 \mathrm{pc}$ ) seem to be too optimistic. In particular, the error bars in the effective temperatures $(1.9 \%$ and $2.4 \%$ for the primary and the secondary, respectively) were crucial to evaluate the error bar in $\alpha_{\mathrm{ov}}$.

In order to try to elucidate this contradictory situation, we use the ratio of effective temperature instead of the effective temperatures themselves to estimate the amount of core 
overshooting in V380 Cyg. This is a better constrained parameter because it is directly derived from the light curves and because it is independent of calibrations, distances, and model atmospheres.

\section{The apsidal-motion rate and the absolute dimensions}

The apsidal-motion rate in V380 Cyg has remained an open question for decades. Because of the long duration of the eclipses, the determination of the times of minima is not straightforward (Guinan et al. 2000). The latter authors derived the most recent apsidal-motion rate for V380 Cyg of $0.0083 \pm 0.0006$ degrees/cycle based on data from Kron (1935), Battistini et al. (1974), Dorren \& Guinan (not published) and Guinan et al. (2000). This value will be adopted in the present work.

The first light curves were not accurate enough and the observations by Kron (1935), Semeniuk (1968) and Battistini et al. (1974) were recently superseded by those performed by Guinan et al. (2000) who used a robotic $25 \mathrm{~cm}$ telescope with an attached $U B V$ photometer, complemented with extra campaigns with an $80 \mathrm{~cm}$ telescope. Given that V380 Cyg is a complex binary, the modelling of the light curves is not simple. In addition to the mentioned problems of the duration of the eclipses, there seems to be an excess in the scatter which may be related to stellar pulsation or to another kind of variability. For an overview of the other peculiarities present in the system, we refer to the quoted paper. Of course, these difficulties do not necessarily imply that acceptable fits using synthetic light curves are impossible to obtain.

The effective temperatures of V380 Cyg are also a matter of discussion. Even if we concentrate our attention only on the last published papers, a clear inconsistency is found. Hill \& Batten (1984) gave the temperatures both at the poles and at the equator, and found a relative difference for the primary of the order of 7\%. The results by Lyubimkov et al. (1996) on the other hand differ by $12 \%$ from those listed by Hill \& Batten. However, the fact that really induced us to consider those determinations of the effective temperatures with caution is that the investigation by Guinan et al. (2000) also indicated large internal discrepancies between the effective temperatures derived from different photometric systems. In addition, even for a given photometric system, differences up to $10 \%$ are found when different calibrations are used. For different photometric systems the disagreements are even larger and can reach up to $18 \%$. In an effort to improve the determination of $T_{\text {eff }}$, Guinan et al. (2000) adjusted Kurucz models (1994) to IUE ultraviolet spectrophotometry, deriving an effective temperature of $21350 \pm 400 \mathrm{~K}$ for the primary. This value is not fully compatible with those derived in the same paper using photometry but the method is nevertheless a step forward.

Concerning the absolute dimensions, Lyubimkov et al. (1996), discussed the evolutionary status of V380 Cyg and found that the masses, as derived from theoretical models, are systematically larger than those obtained from observations. A similar effect was also found for $\alpha$ Vir (Lyubimkov et al. 1995). This behaviour may in fact be part of a more serious problem
Table 1. Relevant astrophysical parameters.

\begin{tabular}{lll}
\hline \hline & Primary & Secondary \\
Mass & $11.1 \pm 0.5$ & $6.95 \pm 0.25$ \\
Radius & $14.7 \pm 0.2$ & $3.74 \pm 0.07$ \\
$\log g$ & $3.148 \pm 0.023$ & $4.133 \pm 0.023$ \\
$\log T_{\text {eff }}$ & $4.329 \pm 0.008$ & $4.312 \pm 0.011$ \\
Effect. Temp. ratio & $0.96 \pm 0.02$ & \\
$\dot{\omega}_{\text {obs }}$ & $0.0083 \pm 0.0006$ & \\
\hline
\end{tabular}

as described in Herrero et al. (1992). Furthermore Lyubimov et al. (1996) pointed out that V380 Cyg is located near the region of pulsational instability typical of $\beta$ Cep type stars. However, contrary to the case of $\alpha$ Vir, no definite pulsational signal was detected so far, though this possibility cannot yet be fully discarded.

Recently, Guinan et al. (2000) presented a new analysis of the photometric and spectroscopic elements of V380 Cyg. The spectroscopic data was taken from Popper \& Guinan (1998) and the light curves were obtained as described above. The astrophysical parameters derived from the analysis of the combination of photometry and spectroscopy are shown in Table 1 and will be adopted in the present work.

\section{The not so clear stellar models for V380 Cyg}

Present day stellar evolution codes still use a considerable number of free parameters, such as the mixing-length scale, the amount of core overshooting, the chemical composition, etc. Core overshooting, e.g., is computed by increasing the size of the convective core given by the classical Schwarzschild criterion by an extra distance $d_{\mathrm{ov}}=\alpha_{\mathrm{ov}} H_{\mathrm{p}}$, where $H_{\mathrm{p}}$ is the pressure scale height and $\alpha_{\mathrm{ov}}$ is a free parameter. Several investigations on DLEBS indicate that a good level of inter-agreement between theory and observations is obtained by considering $\alpha_{\mathrm{ov}}$ between 0.2-0.3 (Claret 1991; Claret \& Giménez 1993; Pols et al. 1997). This interval is also confirmed by more recent studies (Claret \& Willems 2002; Lastennet \& Valls-Gabaud 2002). However, for the specific case of V380 Cyg this range of core-overshooting parameters interval seems to be insufficient to produce an acceptable fit to the observations (Claret 1991; Claret \& Giménez 1993; Lyubimkov et al. 1996; Guinan et al. 2000). In fact, Claret (1991) invoked the possibility that a larger $\alpha_{\text {ov }}$ could explain the absolute dimensions as well as the apsidal motion rate but the large uncertainties in the absolute dimensions at that time did not allow a more detailed study.

In order to test the influence of $\alpha_{\mathrm{ov}}$ on the comparison between the theoretical predictions and the observationally determined absolute dimensions and the apsidal-motion rate of V380 Cyg, we have computed several stellar models adopting different values of $\alpha_{\text {ov }}$ for the observed masses of both components of V380 Cyg, with their respective errors. To this end, we used a modified version of the code described in Claret (1995). The adopted metallicity was $Z=0.012$, which is compatible with the observational determination of the metallicity. 
For the helium content we adopted the metallic enrichment law derived by Claret \& Willems (2002), which gives $Y=$ 0.261 . Small variations around the adopted chemical composition have no significant effect on our final conclusions. The observational error bars on the effective temperatures of the components of V380 Cyg (1.9\% and $2.4 \%$ for the primary and the secondary, respectively) seems to be too optimistic though, as well as the distance determination of $1000 \pm 40 \mathrm{pc}$. In order to support this, we recall that the components are rotationally and tidally distorted (more at the periastron), so that a clear definition of $T_{\text {eff }}$ and $\log g$ is not a secure question. In addition, the observational errors and the intrinsic uncertainties in the atmosphere model fit (the opacities, the plane parallel approximation, the chemical composition, the adopted extinction law, and in the case of the secondary the low luminosity ratio), in our opinion, do not permit such an accurate determination of $T_{\text {eff }}$. Due to these limitations, we decided to use the effective temperature ratio $\mathrm{TR}=T_{\mathrm{eff} 2} / T_{\mathrm{eff} 1}$ instead of their absolute values since this parameter is determined much more accurately from light curve analyses. A similar procedure was used previously by Claret \& Willems (2002) in order to avoid the problems resulting from calibration/model dependencies. The results are shown in Figs. $1-3$ for $\alpha_{\text {ov }}=0.0$ up to $\alpha_{\text {ov }}=0.8$.

Let us first inspect the case of the standard models with $\alpha_{\mathrm{ov}}=0.0$ (Fig. 1). As usual, we adopt as a common age of the system the point where the theoretical effective temperature ratio and $\log g$ are intersected simultaneously by the lines representing the respective observational values. If we inspect the first two panels of Fig. 1 around the age of $17.35 \mathrm{Myr}$, we note that the values of TR and $\log g$ are in good agreement with the theoretical predictions, though the uncertainty in the age of the secondary star is obvious. This is not surprising since we are simultaneously dealing with a star near the ZAMS and a much more evolved star. The uncertainty is therefore a direct consequence of the low mass ratio $q$ and of the difficulty to derive ages for young components. Furthermore we computed the time evolution of the theoretical mean apsidal-motion constant $\overline{k_{2}}$ using the known formulae and derived the related astrophysical parameters directly from the corresponding evolutionary track. The relativistic contribution is around $12 \%$ of the total and was conveniently taken into account. We note that the theoretical evolution of $\overline{k_{2}}$ as shown in Figs. 1-3 is not necessarily correct since the secular changes in the period and the eccentricity are not properly taken into account, i.e. $P$ and $e$ are kept constant at their actual observational values. However, since the contribution of the secondary to $\dot{\omega}$ is almost negligible, $\bar{k}_{2}$ is essentially dominated by the apsidal-motion constant of the primary, so that $\bar{k}_{2} \approx k_{21}$, irrespective of the values of the period and the eccentricity. At the same isochrone as before, the average value of $\overline{k_{2}}$ to be compared with the observed apsidal-motion rate is then given by $\log \bar{k}_{2 \mathrm{obs}} \approx-2.90$. The smallest value reached by the theoretical value of $\log \bar{k}_{2}$ is of the order of $-2.70 /-2.75$ indicating that these models pass the TR and the surface gravity tests, but fail in the internal structure constants diagnostics.

Figure 2 illustrates the same as Fig. 1, but for $\alpha_{\mathrm{ov}}=0.2$. The models corresponding to the masses affected by the respective error bars are also plotted in order to estimate the errors in
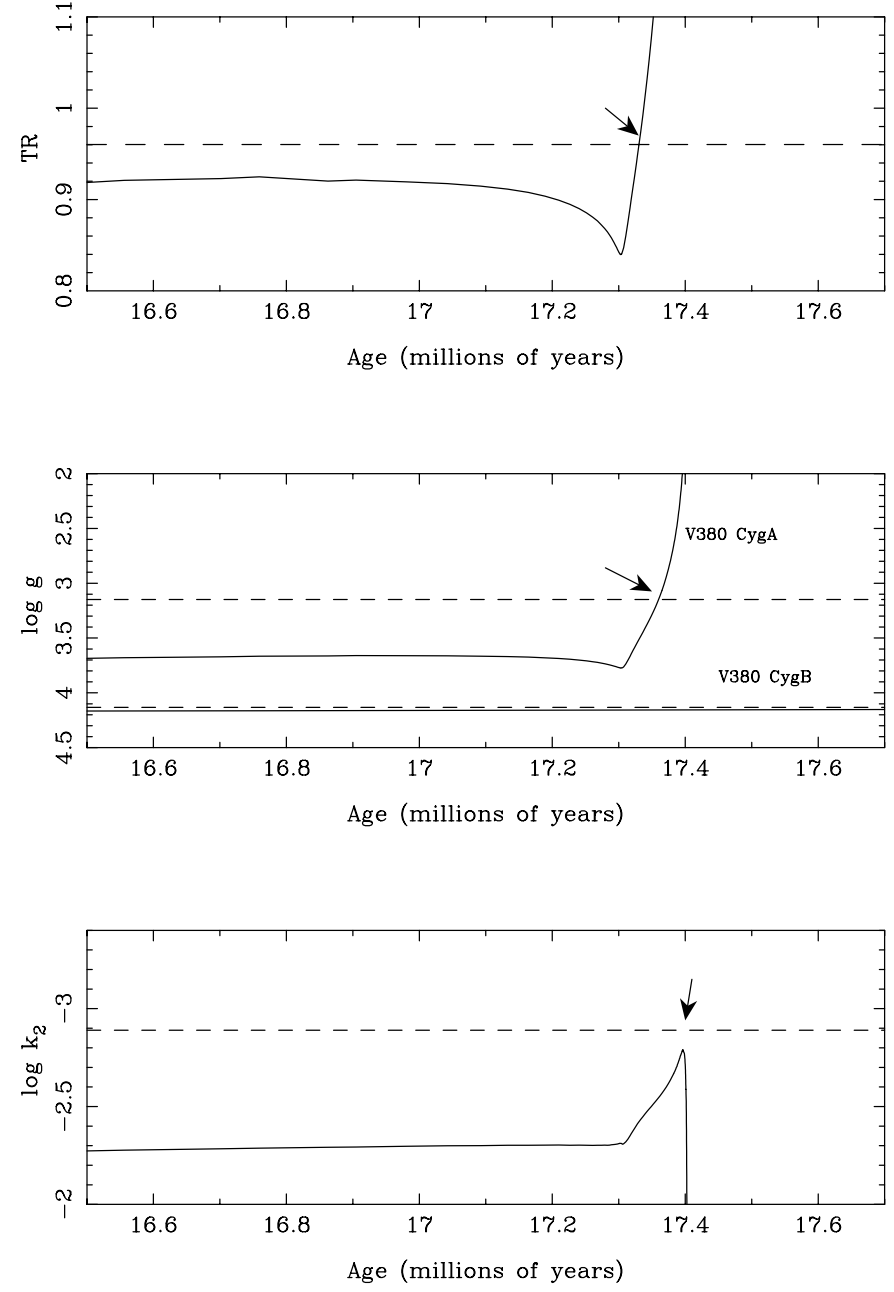

Fig. 1. The theoretical ratio of the effective temperatures (first panel), the surface gravity (second panel) and the mean apsidal-motion constant $\overline{k_{2}}$ (third panel) as a function of age (in millions of years). The solid and dashed lines denote the theoretical and the observed values, respectively. Case $\alpha_{\mathrm{ov}}=0.0, X=0.727, Z=0.012$.

the determination of the age of the system. They confirm, once again, that the uncertainty in the age of the secondary is not small and that no unambiguous discrimination between the different models for this star is possible. The mean errors in the age of the primary are of the order of $\pm 1.5 \mathrm{Myr}$. Let us examine now the behaviour of the average internal structure constant. As core overshooting increases the stellar mass concentration, a pronounced decrease in $\overline{k_{2}}$ compared with the previous figure can be seen in the third panel of Fig. 2. It is noticeable that at the same isochrone, the models corresponding to the observed masses are able to reproduce the TR and radii as well as the apsidal-motion rate. As in the previous case, the models indicate that the primary is in a very fast transitional phase of stellar evolution showing rapid changes in the radius. Similar effects are obtained as the amount of core overshooting is increased even further. For illustration, the results for $\alpha_{\mathrm{ov}}=0.4$ and $\alpha_{\mathrm{ov}}=0.6$ are shown in the third panel of Fig. 3 . 

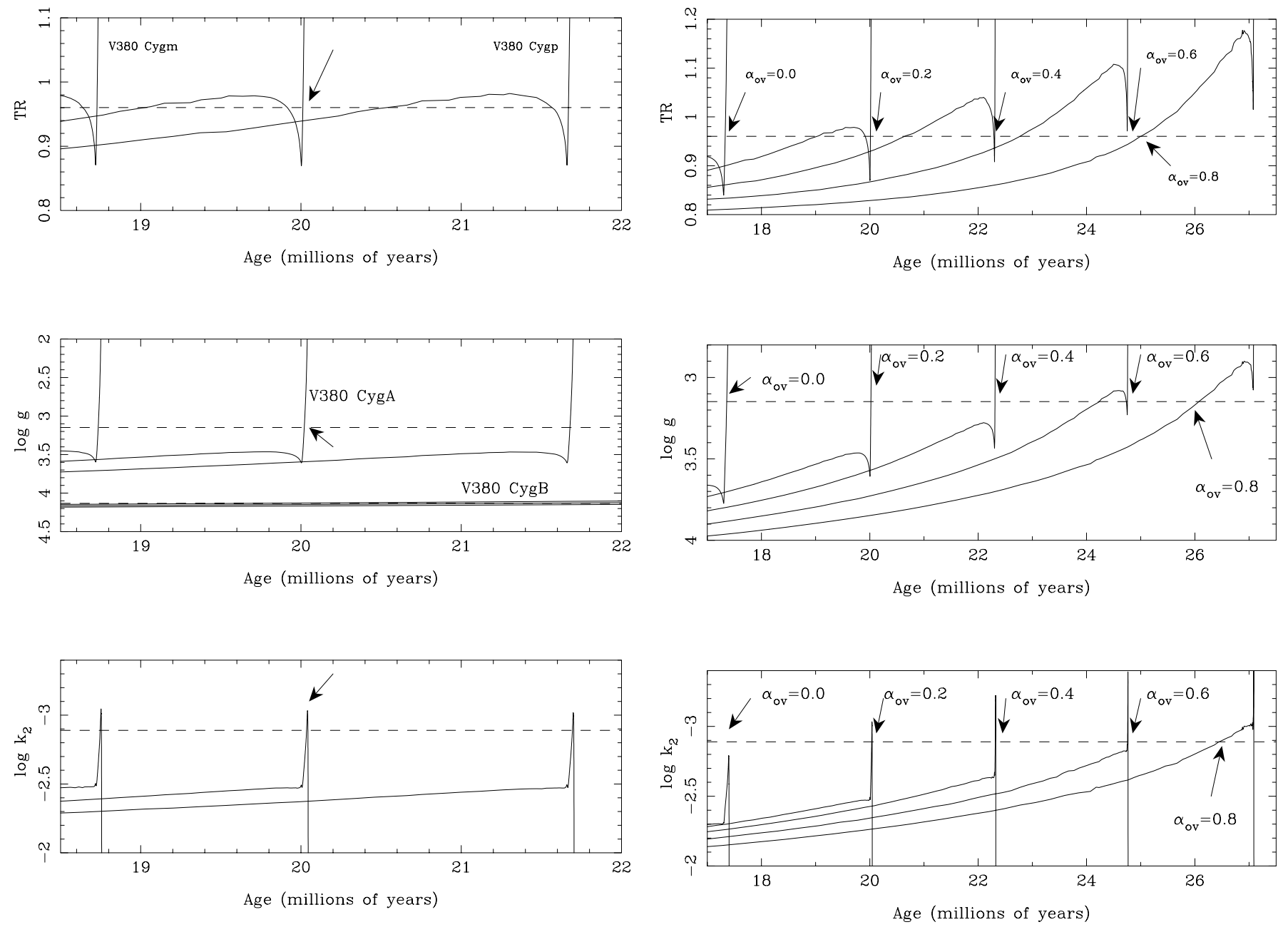

Fig. 2. The same as Fig. 1, but with $\alpha_{\mathrm{ov}}=0.2$. The curves labelled with V380 Cygm and V380 Cygp represent the models for the primary affected by the observational errors in the masses, while the central curve denotes the models computing using the observed masses. The models for the secondary are practically indistinguishable in this interval.

\section{Consequences: The not so definitive conclusions}

Let us summarize our results and add the case $\alpha_{\mathrm{ov}}=0.8$ to the models represented in Figs. 1-2. The complete series of models is displayed in Fig. 3. The models for the secondary were not represented for the sake of clarity. For an acceptable common age for each case (indicated by the arrows) all models for the primary suggest that it is in a critical phase of evolution, with the exception of the models with $\alpha_{\mathrm{ov}}=0.8$. The latter models indicate that the primary is still on the Main Sequence though it already burnt about $90 \%$ of its initial hydrogen content. Inspection of Fig. 3 shows that not only the radius changes quickly around the point where the theoretical values of TR and $\log g$ cross the dashed line, but that the corresponding values of $\overline{k_{2}}$ are also rapidly changing functions of time in the same interval, which has direct consequences for the interpretation of the apsidal-motion rate of V380 Cyg. In contrast to usual thinking, not only models with a large value of $\alpha_{\text {ov }}$ are able to produce satisfactory fits for the absolute dimensions and

Fig. 3. A summary of the results. The models are labelled following their values of $\alpha_{\mathrm{ov}}$ from 0 up to 0.8 . The models for the secondaries are not plotted for the sake of clarity. Note that all models which share a common age are very near of their critical point of evolution, except those for $\alpha_{\mathrm{ov}}=0.8$.

the apsidal-motion rate of V380 Cyg. The system is currently in such a critical phase that even small errors in the age imply large uncertainties in the radius and in $k_{2}$. Consequently, not only the solution associated with $\alpha_{\mathrm{ov}}=0.6$ can be considered as compatible with the observations but also the solutions associated with $\alpha_{\mathrm{ov}}=0.2$ and $\alpha_{\mathrm{ov}}=0.4$. When $\alpha_{\mathrm{ov}}$ is increased up to 0.8 , which locates the primary on the Main Sequence, it is no longer possible to find an acceptable common age, and the fit becomes worse. One could argue that the same effect could occur for other DLEBS and in particular for those showing apsidal motion. However, the question is then whether or not they are near the TR-radius- $k_{2}$ transition phase and whether their secondaries serve as good discriminators. The third panel of Fig. 2 is useful to understand this question: the observational errors in the masses yield an error in the theoretical $\overline{k_{2}}$ which is large because the internal concentration constant is a rapidly changing function of time and mass in the observed interval of the surface gravity. For a given mass, the effect of core overshooting is more pronounced as the star evolves, so that one might expect more evolved systems to be more suitable to test 
the extra mixing through apsidal-motion test. The high rate of variation of the theoretical $k_{2}$ is critical in those phases. Such a test could therefore only be carried out reliably for evolved systems if the masses were known with extreme accuracy. Within the current level of our observational and theoretical knowledge of V380 Cyg, the apsidal-motion test is therefore not a definitive test for the internal structure of the components of this system.

The results we have obtained imply that the error bars on $\alpha_{\mathrm{ov}}$ as determined by Guinan et al. (2000) should be revised. As we have seen, more than one value of $\alpha_{\mathrm{ov}}$ is compatible with observations if one uses the effective temperature ratio instead of the effective temperatures themselves. From the comparison between the observational data and the properties of the stellar models, we derive $\alpha_{\mathrm{ov}}=0.6_{-0.4}^{+0.1}$. However, we have some reserve about this value of $\alpha_{\mathrm{ov}}$. As far we know, there is no independent support for it neither from ColourMagnitude Diagrams of clusters nor from DLEBS (Claret \& Willems 2002). It is therefore possible that it could be a spurious solution, though within the uncertainties, it may also really indicate that a larger value of $\alpha_{\mathrm{ov}}$ is necessary to fit more massive stars. Apparently, the new error bars do not seem to be so important. However, it is important to determine the possibility of a hypothetical dependency of $\alpha_{\mathrm{ov}}$ on the stellar mass (Claret 1991; Ribas et al. 2000). Using the newly established value, the dependency, if exists, is less pronounced and uncertain than previous investigations suggested. The use of the effective temperatures and their respective observational error bars should lead to more refined solutions. However, as mentioned, these error bars seem to be too optimistic and the results based on them should be taken with caution. The uncertainty indicated for $\alpha_{\mathrm{ov}}$ should not be interpretated as a negative result or a backward step. Instead, it indicates that error in the core-overshooting parameter cannot be determined with a precision of 0.1 using realistic observational errors as a constraint, mainly when dealing with an EBS in a very critical phase of evolution.

The derived value of $\alpha_{\mathrm{ov}}$ may be somewhat modified if rotation is considered, taking into account the induced-mixing inestabilities as well as the transport of angular momentum. This work is currently being carried out and we expect to present the results in a future paper.

As we have seen, the contribution to the total apsidalmotion rate of the secondary is much smaller than the contribution provided by the primary since the ratio $c_{21} / c_{22}$ is very large. This means that contrary to usual investigations of DLEBS, we are essentially testing only the primary component. Hence questions like what is the role of the mass ratio in the evolutionary path of binary stars; why do some binaries present low values of $q$, are close binaries with low $q$ values really coeval and is the chemical composition really equally distributed between such components need yet to be answered. As a simple speculation, we think that such a common evolution should be rather competitive and possibly the answer to the last two questions would be negative. This concept of competitive evolution is hard to define, though it is more evident in the case of interacting binaries given that it is not easy to search for a conspicuous example in binaries for which contact of the star with its Roche lobe is not yet achieved. However, if we examine a random sample of binaries with good determinations of the absolute dimensions, the worst adjustments with stellar models often correspond to binaries with low $q$ values. Should these discrepancies then be related to deficiencies in stellar modelling and selection effects? Surely the answer is yes, but we believe this only partly explains the problem. Only when we obtain more reliable information on the role (if possible) of sharing the primordial matter between the components of close binaries with low $q$, can the answer to the question be more realistically quantified.

Acknowledgements. The Spanish DGI (project AYA2000-1559) is acknowledged for the support received. This research has made use of the Simbad database, operated at CDS, Strasbourg, France.

\section{References}

Alexander, D. R., \& Ferguson, J. W. 1994, ApJ, 437, 879

Battistini, P., Bonifazi, A., \& Guarnieri, A. 1974, Ap\&SS, 30, 163

Caughlan, G. R., \& Fowler, W. A. 1988, Atomic Data Nucl. Data Tables, 40, 283

Claret, A. 1991, Ph.D. Thesis, Universidad de Granada

Claret, A. 1995, A\&AS, 109, 441

Claret, A., \& Giménez, A. 1993, A\&A, 277, 487

Claret, A., \& Willems, B. 2002, A\&A, 388, 518

Guinan, E, Ribas, I., Fitzpatrick, E. L., et al. 2000, ApJ, 544, 409

Herrero, A., Kudritzki, R. P., \& Vilchez, J. M. 1992, A\&A, 261, 209

Hill, G., \& Batten, A. H. 1984, A\&A, 141, 39

Huebner, W. F., Merts, A. L., Magee, N. H., \& Argo, M. F. 1977, Astrophys. Opacity Library, UC-34b

Iglesias, C. A., \& Rogers, F. J. 1996, ApJ, 459, L35

Kron, G. E. 1935, ApJ, 82, 225

Kurucz, R. L. 1994, CD-ROM collection, number 19

Lastennet, E., \& Valls-Gabaud, D. 2002, A\&A, 396, 551

Lyubimkov, L. S., Rachkovskaya, T. M., Rostopchin, S. I., \& Tarasov, A. E. 1995, Astron. Rep., 39, 186

Lyubimkov, L. S., Rachkovskaya, T. M., Rostopchin, S. I., \& Tarasov, A. E. 1996, Astron. Rep., 40, 46

Pols, O. R., Schröder, K.-P., Eggleton, P. P., \& Manners, J. 1997, MNRAS, 289, 869

Popper, D. M., \& Guinan, E. F. 1998, PASP, 110, 572

Ribas, I., Jordi, C., \& Giménez, A. 2000, MNRAS, 318, L55

Semeniuk, I. 1968, Acta Astron., 18, 1 